\title{
A rare case of obstructive azoospermia due to compression of the seminal vesicle and ejaculatory duct by a large lower ureteric stone
}

\author{
Priyadarshi Ranjan ${ }^{1}$, MS, MCh, Abhishek $\underline{\text { Yadav }}^{1}$, MBBS, MS, Rohit Kapoor ${ }^{1}$, MBBS, Ranjana Singh ${ }^{2}$, MBBS, MHA
}

\begin{abstract}
Male infertility due to obstructive azoospermia is a well-known entity. It is characterised by obstruction to the outflow of sperms either in the epididymis, vas, seminal vesicles or the ejaculatory ducts. We describe a rare case of obstructive azoospermia due to compression of the ejaculatory duct and seminal vesicle by a large lower ureteric stone in a 30-year-old man who had infertility for the past ten years. The patient's azoospermia resolved after removal of the stone.
\end{abstract}

Keywords: ejaculatory duct, male infertility, obstructive azoospermia, seminal vesicle, stone

\section{INTRODUCTION}

Obstructive azoospermia can occur due to numerous causes, with the most common site of obstruction being the epididymis. Extraluminal obstruction mostly occurs near the ejaculatory duct or seminal vesicles, usually due to ductal stenosis, prostatic cysts or rarely, large lymph nodes. A large lower ureteral diverticulum, with a stone causing seminal vesicular compression and azoospermia, has not been previously described. We present the case of an infertile man whose azoospermia resolved after removal of the ureteric stone.

\section{CASE REPORT}

A 30-year-old Indian man who had primary infertility for 12 years presented with complaints of occasional left flank pain and fever. He had intermittent episodes of turbid urine over the last eight years and low ejaculatory volume since puberty. The patient had been evaluated for primary infertility in other peripheral centres before presenting at our centre for recurrent flank pain and dysuria. He was a nonsmoker and had no history of alcohol intake or use of any gonadotoxic medication. On examination, mild left flank tenderness was present. Genital examination was unremarkable. Digital rectal examination revealed a normal-sized prostate with a stony, hard mass (measuring $2.5 \mathrm{~cm} \times 2.5 \mathrm{~cm}$ ) that was palpable above the prostate on the left side and crossing over the midline.

The patient was worked up for infertility; and his previous semen analyses had revealed azoospermia on three successive occasions. Serum follicle stimulating hormone and testosterone values were within normal limits. Testicular fine needle aspiration suggested adequate spermatogenesis. Plain abdominal skiagram showed a large radiopaque shadow over the left pubic tubercle (Fig. 1). Abdominal ultrasonography revealed gross left hydroureteronephrosis up to the lower ureter, along with paper-thin parenchyma. There was a negative acoustic shadow in the area of the lower ureter that was suggestive of a calculus; however, the site of origin was not delineated. Transrectal ultrasonography (TRUS) suggested a large echogenic shadow in or around the left seminal vesicle, leading to obstruction of the ejaculatory ducts with resultant dilatation of both the seminal vesicle and vas. Thus, TRUS raised the possibility of a seminal vesicle stone rather than a ureteric stone. Unfortunately, no image of the TRUS finding is available. Contrast-enhanced computed tomography (CECT) imaging revealed a calculus in the left seminal vesicle region with gross left hydroureteronephrosis (Fig. 1). Subsequent renal diuretic imaging revealed negligible function of the left kidney and a normal right kidney.

We were faced with a radiological dilemma, as the location of the stone, whether in the seminal vesicle or the lower ureter, was not determined. Finally, a left retrograde pyelogram (Fig. 1) was performed, which confirmed the presence of a stone measuring $3 \mathrm{~cm}$ in the large lower ureteral diverticulum. The ureteral diverticulum with the stone in the left lower ureter was causing proximal hydroureteronephrosis, which may have also contributed to the obstruction of the ejaculatory duct and seminal vesicle. The patient was managed with laparoscopic nephroureterectomy and had an uneventful recovery.

Follow-up semen analysis three months later revealed a sperm count of $10 \mathrm{million} / \mathrm{mL}$ in the ejaculate. A subsequent semen analysis showed oligoasthenospermia, with a sperm count of $15 \mathrm{million} / \mathrm{mL}$ and a progressive motility rate of $10 \%$. However, the patient's partner was still unable to achieve pregnancy at six months of follow-up. Two subsequent semen analyses also confirmed the presence of sperm in the ejaculate, with a sperm count of 20 million $/ \mathrm{mL}$.

\footnotetext{
${ }^{1}$ Department of Urology and Kidney Transplantation, Sanjay Gandhi Post Graduate Institute of Medical Sciences, Lucknow, ${ }^{2}$ Department of Hospital Administration, Post Graduate Institute of Medical Education and Research, Chandigarh, India

Correspondence: Dr Priyadarshi Ranjan, Assistant Professor, Department of Urology and Kidney Transplantation, Sanjay Gandhi Post Graduate Institute of Medical Sciences, Lucknow, Uttar Pradesh, India. priydarshiranjan@sify.com
} 

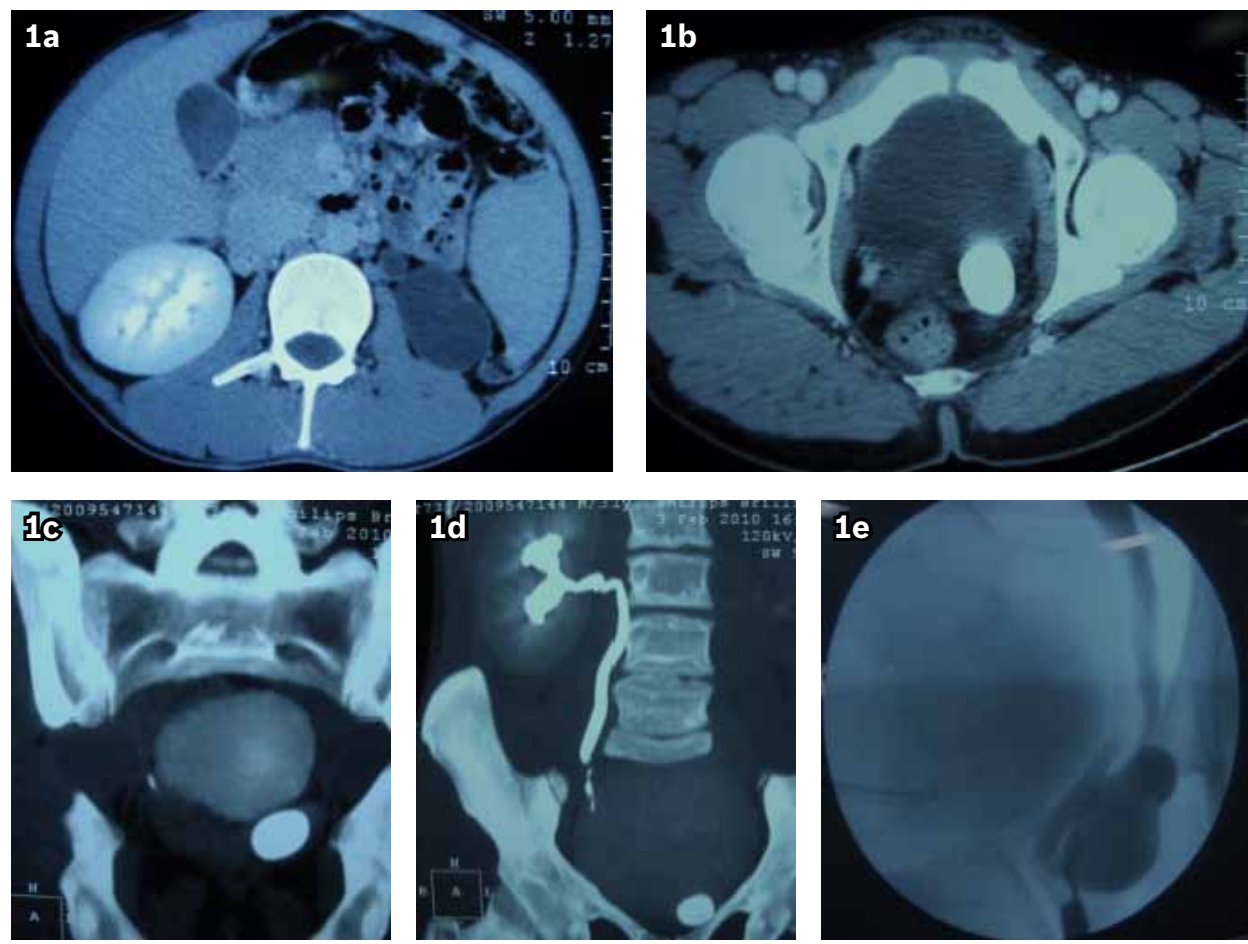

Fig. 1 CT images show (a) left gross hydroureteronephrosis and poorly functioning left kidney as a result of obstruction due to a lower ureteric stone; and (b) the culprit lower ureteric stone in the diverticulum. (c) Axial CT image shows the culprit lower ureteric stone in the diverticulum. (d) CT urogram shows nonfunctioning left kidney. (e) Retrograde pyelogram shows the large ureteral diverticulum and the stone responsible for seminal vesicle and ejaculatory duct compression.

\section{DISCUSSION}

The finding of a ureteral diverticulum is uncommon, with less than 100 cases reported. Giant ureteral diverticula $(>3 \mathrm{~cm})$ containing stones are extremely rare and have not been known to account for compression of the seminal vesicle and ejaculatory ducts, leading to infertility.

Ureteral diverticula were first classified by Culp into two categories, congenital and acquired.(1) Congenital ureteral diverticula occur as a result of an aberrant development of the ureteric bud before it reaches the metanephric tissue. They are characterised as single, dilated blind-ending branches of a bifid ureter, made up of an outpouching of all layers from a normal ureteral wall, and are often more than $0.5 \mathrm{~cm}$ in diameter. They can either be asymptomatic or present with recurrent urinary tract infections in males. However, they have not been described to cause obstructive azoospermia. Acquired (false) ureteral diverticula, on the other hand, are mucosal protrusions through a defect in the ureteral wall. These are usually related to traumatic instrumentation, surgery or rupture of stone, with obstruction. They are mostly single and larger than pseudodiverticula, as defined by Culp.(1) The aetiology of true ureteral diverticulum remains obscure, although a number of factors have been suggested. Barrett and Malek, who studied 12 patients with ureteral diverticulum over a period of 25 years, reported that associated renal or ureteral diseases may potentiate the development of ureteral diverticulum. Some of these patients had chronic infection, vesicoureteral reflux or stones, and others had bladder outlet obstruction. Most cases of ureteral diverticulum in their series

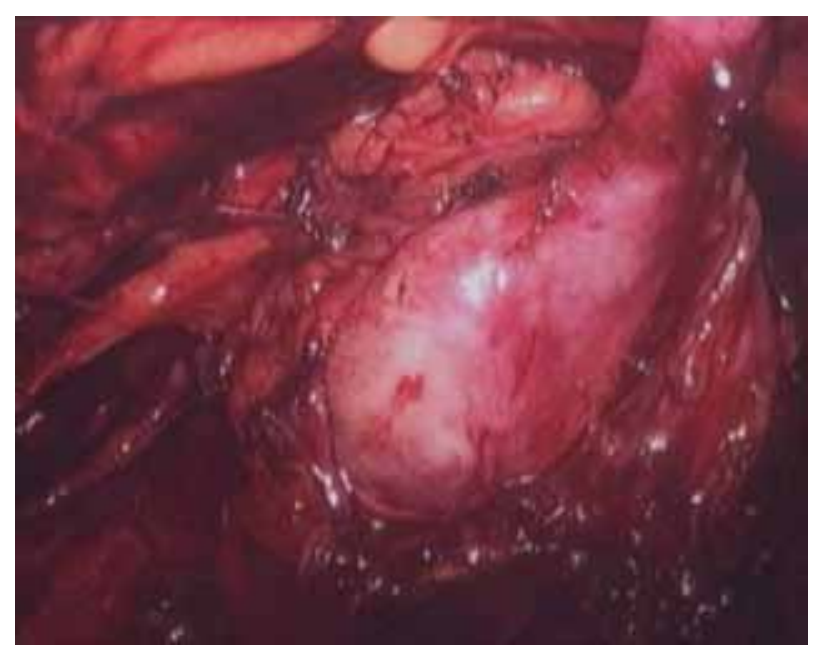

Fig. 2 Intraoperative photograph shows the giant ureteral diverticulum and ureteric stone after dissection near the left vesicoureteric junction.

occurred at the level of the vesicoureteric junction. ${ }^{(2)}$ Rank et al considered ureteral diverticula to be merely overextended bifid ureters, ${ }^{(3)}$ while Franko et al opined that firbroepithelial polyps and congenital diverticula of the ureter are part of a spectrum of the same developmental anomaly.(4)

Our patient presented with considerable diagnostic difficulty, as the CECT image was consistent with a calculus in the left seminal vesicle area with gross left hydroureteronephrosis and poorly functioning left kidney. TRUS suggested the presence of a large calculus in or around the left seminal vesicle, leading to ejaculatory duct obstruction with resultant dilatation of both 
the seminal vesicle and vas. In our patient, the presentation was primary azoospermia and recurrent left flank pain. We propose that this acquired distal ureteral diverticulum may be a sequel of gradual obstruction of the distal ureter by the stone, leading to repeated infections and enlargement of the diverticula, which caused compression of both the ejaculatory duct and seminal vesicle, as well as gradually declining left renal function. Although contributory, infection occurs only after stasis due to stones. It is very unlikely that obstructive azoospermia can occur primarily due to recurrent infection or inflammation. The mechanism in this case of acquired diverticula is similar to that reported by Barrett and Malek.(2) Imaging studies are important for the detection of the lesion as well as in determining its nature, although it may be difficult to establish the site of origin using noninterventional imaging.
When a ureteral diverticulum is diagnosed, therapy depends on the patient's clinical presentation. It may be managed by releasing the obstruction of the collecting system and creating a patent ureter with ureteroscopic measures, laparoscopic excision or open surgery. A nephrectomy should only be performed on infected or nonfunctioning kidneys. Careful evaluation of the upper urinary tracts to exclude uncommon causes should also be done in patients with obstructive azoospermia.

\section{REFERENCES}

1. Culp OS. Ureteral diverticulum classification of the literature and report of an authentic case. J Urol 1947; 58:309-21.

2. Barrett DM, Mallek RS. Ureteral diverticulum. J Urol 1975; 114:33-5.

3. Rank WB, Mellinger GT, Spiro E. Ureteral diverticula: etiologic considerations. J Urol 1960; 83:566-9.

4. Franco I, Choudhury M, Eshghi M, Bhalodi A, Addonizio JC. Fibroepithelial polyp associated with congenital ureteral diverticulum: report of 2 cases. J Urol 1988; 140:598-600. 